\title{
Influence of water soluble carbon dots on the growth of wheat plant
}

\author{
Shweta Tripathi $\cdot$ Sabyasachi Sarkar
}

Received: 31 July 2014/ Accepted: 27 August 2014/Published online: 9 September 2014

(C) The Author(s) 2014. This article is published with open access at Springerlink.com

\begin{abstract}
The effect of water soluble carbon nano-dots (wsCND) on the growth of root and shoot of wheat plants under light and dark conditions has been studied. The wsCND enhances the growth of root and shoot both in light and dark conditions. The effect of wsCND on the growth of root was relatively more compared to that of shoot. Scanning electron and Fluorescence microscopic analysis show that wsCNDs enter inside the plant. Raman spectroscopy also confirms this. The present study shows that wsCNDs are non-toxic to the wheat plant and can be used to enhance the production of this cereal crop.
\end{abstract}

Keywords Plant $\cdot$ Carbon nano-dots $\cdot$ Growth

\section{Introduction}

Over last few years, there have been extensive studies to understand the influence of nanomaterials on the growth of different types of plants (Khodakovskaya et al. 2009; Khodakovskaya et al. 2013a, b; Lu et al. 2002; Cañas et al. 2008; Yang et al. 2006; Hong et al. 2005a, b; Tan et al. 2009; Yuan et al. 2011; Khodakovskaya et al. 2013a, b; Mahajan et al. 2011; Wang et al. 2012; Larue et al. 2012; Miralles et al. 2012; Feizi et al. 2012; Tripathi et al. 2011; Sonkar et al. 2012). Khodakovskaya et al. (2009, 2013a, b)

\section{S. Tripathi}

Indian Institute of Science Education and Research Kolkata,

Mohanpur 741252, India

\section{S. Sarkar $(\bowtie)$}

Indian Institute of Engineering Science and Technology,

Shibpur, Howrah 711103, India

e-mail: abya@iitk.ac.in; protozyme@gmail.com demonstrated that at very low doses MWCNT can penetrate the seed coat and enhance the germination of tomato plant. Lu et al. (2002) studied on soya bean and found that the mixture of nano- $\mathrm{SiO}_{2}$ and nano- $\mathrm{TiO}_{2}$ increases nitrate reductase which causes seed germination. Canas et al. (2008) worked on onion and cucumber and found that functionalized carbon nanotubes enhance root elongation. Yang et al. (2006) and Hong et al. (2005a, b) studied the growth of the spinach and found that proper concentration of $\mathrm{TiO}_{2}$ could enhance photosynthesis and nitrogen metabolism. Tan et al. (2009) demonstrated the use of MWCNT to increase reactive oxygen species (ROS) and decrease the cell viability of rice plant. Yuan et al. (2011) found that SWNT $(50 \mu \mathrm{g} / \mathrm{ml})$ stimulate the growth of mesophyll cells of Arabidopsis. Khodakovskaya et al. (2013a, b) studied at cellular level and showed the positive effect of MWCNT and explained the mechanism for the plant growth in tobacco cells. Mahajan et al. studied mung and Gram seed using nano- $\mathrm{ZnO}$ particle by plate agar method and found that the growth of mung and gram is different at different concentrations. The seedling growth was maximum at $20 \mathrm{ppm}$ for mung and at $1 \mathrm{ppm}$ for gram (Mahajan et al. 2011). Wang et al. (2012) carried out experiment on wheat and found that MWCNT significantly promote cell elongation and increases dehydrogenase activity. Laure et al. (2012) used ${ }^{14} \mathrm{C}$ radiolabelled MWCNT to study wheat and rape-seed quantitatively in hydroponic solution and found that there is no effect on seed germination, root elongation and on dry mass. Miralles et al. (2012) studied the phytotoxicity of wheat and alfalfa using industrial CNT and catalytic impure CNT and found that there was no toxic effect on germination under high concentration. Feizi et al. (2012) found the positive effect on the germination and seedling of wheat when treated with different concentrations of bulk and

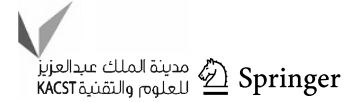


nanosized $\mathrm{TiO}_{2}$. Recently, we reported a study on dicotyledonous plant gram (Cicer arientinum) using water soluble CNT which was synthesized from Mustard oil soot and thus did not contain any trace amount metal impurity and found that it enhances the growth of the gram plant (Tripathi et al. 2011). In a subsequent study, Sonkar et al. (2012) showed that water soluble carbon nano-onions from wood wool also enhance the growth of gram plant. These studies show that carbon nanomaterials like MWCNT, SWCNT enhance growth in plants like dicotyledonous but metallic nano materials show concentration dependent growth in dicotyledonous plant. Dicotyledonous and monocotyledonous plants differ morphologically as they have different arrangements in their vascular bundle, and in their root system. Furthermore, in all monocotyledonous plants, the presence of certain plastid inclusion helps to enhance the photosynthetic process. Therefore, here, we report a detailed study showing the effect of water soluble CND on monocotyledonous plants under light and dark conditions.

\section{Experimental}

\section{Materials}

All reagents were of analytical grade and were purchased from Sigma Aldrich.

\section{Synthesis of water soluble carbon dots}

The water soluble CND was synthesized by the method reported in references Tripathi et al. (2011) and Sonkar et al. (2012). We used mustard oil lamp and collected the carbon soot on the top of its flame on a glass slide. Then soot was washed repeatedly with toluene, alcohol and acetone and finally with water using Soxhlet extractor technique to remove un-burnt oil, poly aromatic hydrocarbon or any other organic molecule produced under such pyrolytic technique. Finally, we slightly modified the procedure as described in reference Tripathi et al. (2011) and this washed and air dried soot was treated with concentrated $\mathrm{HNO}_{3}$ acid contrary to the dilute nitric acid treatment described in reference Tripathi et al. (2011) in cold condition to allow the surface oxidation of the soot (with the evolution of brown nitrogen oxide fumes) and finally that subsided. The soot with the acid was allowed to stand in the boiling water bath to evaporate all the nitric acid. The dry mass was diluted with distilled water and the mixture was evaporated to dryness on a boiling water bath. This process was repeated several times till the final solution became nitrate ion free (tested by Greiss's reagent with zinc powder to reduce nitrate to nitrite).
Finally, the nitrate-free black slurry was warmed to dissolve the soluble part, filtered from the undissolved carbon and the filtrate was dried on the water bath to yield water soluble carbon nano-dots (wsCND). It is noticed that the carbon nano tubes which are formed under dilute nitric acid treatment get fragmented to wsCND under strong oxidative treatment of concentrated nitric acid. The detailed characterization of such carbon dot has already reported by Ghosh et al. (2011) $150 \mathrm{mg}$ of the prepared wsCND was sonicated in water for couple of minutes and it was diluted to one liter. We used this as a stock solution $(150 \mathrm{mg} / \mathrm{L})$ in all the experiments.

\section{Seeds and germination}

Experiments were carried out on the monocotyledonous seeds of wheat. These seeds were surface sterilized using $70 \%$ ethanol and then these were soaked in the water. Seeds took 3-4 days for germination.

\section{Sample preparation for SEM}

Wheat roots were first cut into pieces and then fixed with $2.5 \%$ glutaraldehyde in $0.1 \mathrm{M}$ cacodylate buffer with $\mathrm{pH} 7.4$ for $6 \mathrm{~h}$. Later, the roots were washed with cacodylate buffer containing $4 \%$ sucrose at $\mathrm{pH} 7.4$, then roots were dehydrated using 30, 50, 70 and $100 \%$ ethanol each for $30 \mathrm{~min}$. Later the roots were dewaxed in $100 \%$ xylene and incubated the dewaxed roots into 50:50 xylene and paraffin. After standing for $30 \mathrm{~min}$, we transferred this in $100 \%$ paraffin for $10 \mathrm{~h}$. After $10 \mathrm{~h}$, thin sections of roots were cut from the embedded sample using a microtome (Leica microtome RM225). Sections were washed with xylene and ethanol, respectively, to remove the excess of wax. SEM images were taken on this sample.

\section{Characterization}

Raman spectra were recorded using a Raman spectrometer of Horiba Jobin-Yvon LabRAM HR-800 Grating: 1,800 g/ $\mathrm{mm}$, Exciting wavelength: $632.8 \mathrm{~nm}$ line of $\mathrm{He}-\mathrm{Ne}$ laser and Raman images was taken by a confocal microscope using software labspec. SUPRA40VP Field emission Scanning Electron Microscope (Carl Zeiss NTS GMBH Oberkochen (Germany) equipped with an energy dispersive X-ray (EDAX) in high vacuum mode operated at $10 \mathrm{kV}$. Fluorescence images were taken by a Leica TCS SP2Fluorescence microscope. For Fluorescence microscopic study, excitation wavelength was in the range of $530-550 \mathrm{~nm}$ and the resulting emission was observed in the wavelength range of $575-625 \mathrm{~nm}$. 


\section{Results and discussions}

Characterisation of wsCND

A representative Raman spectrum of the sample is shown in Fig. 1a. There are three dominant peaks in the Raman spectrum at 1,332, 1,594 and 2,680 $\mathrm{cm}^{-1}$ which correspond to the disorder (D) mode, graphite $(\mathrm{G})$ mode and second order harmonics of the D band, respectively. The Raman spectrum clearly indicates the presence of defects in nanoparticles due to the presence of $1,332 \mathrm{~cm}^{-1}$ peaks which could be due to sp (Lu et al. 2002) hybridization related to the oxidation of the wsCND. Functional group in wsCND was characterized by FTIR technique. The FTIR spectrum of the sample is shown in Fig. 1b. The peak around $1,700 \mathrm{~cm}^{-1}$ is for $v(\mathrm{C}=\mathrm{O})$ and the broad band $\sim 3,400 \mathrm{~cm}^{-1}$ showed the presence of $-\mathrm{OH}$ group originated from the carboxylic acid groups as well as the $\mathrm{C}-\mathrm{OH}$ groups present in the wsCND. The broad and high intensity peak around $1,250 \mathrm{~cm}^{-1}$ is due to $\mathrm{C}-\mathrm{O}$ from the $\mathrm{C}-\mathrm{OH}$ groups. In addition, the peak around
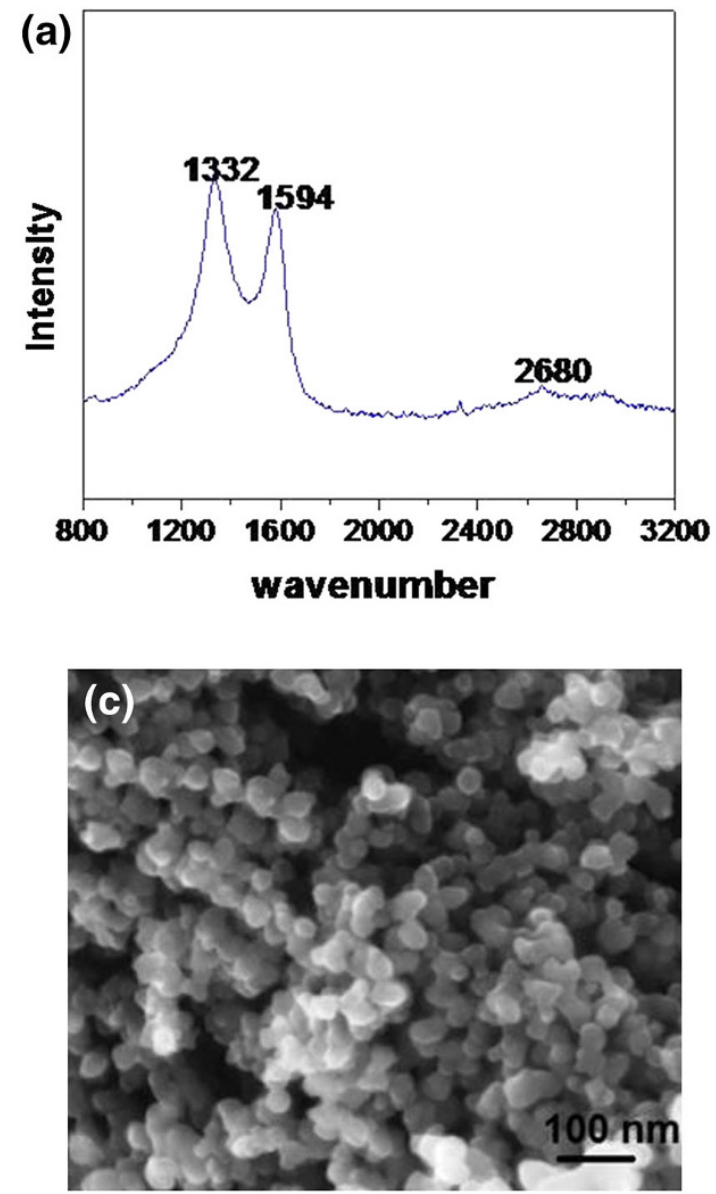

$1,600 \mathrm{~cm}^{-1}$ may be mixed with $\mathrm{C}=\mathrm{C}$ vibration along with some water due to its $\sigma\left(\mathrm{H}_{2} \mathrm{O}\right)$ vibration. The shape and the size of wsCND were characterized by scanning electron microscopy (SEM). The SEM image of the sample is shown in Fig. 1c. It is clear from this image that the size of the particles ranges between 20 and $100 \mathrm{~nm}$ and they seem to be spherical in shape. EDX analysis of nanoparticles was carried out to find out the presence of different elements in the system. A plot of EDX analysis is shown in Fig. 1d. It is clear from this plot that the major components of the nanoparticles are carbon with a minor part of oxygen. All these characterization clearly established that these nanoparticles are made up of carbon atoms along with oxygen functionality commonly from carboxylate and hydroxyl groups which make the nanoparticle water soluble.

Growth of wheat plants with wsCND

To understand the influence of wsCND on the growth of wheat plant under light and dark conditions, wheat plants
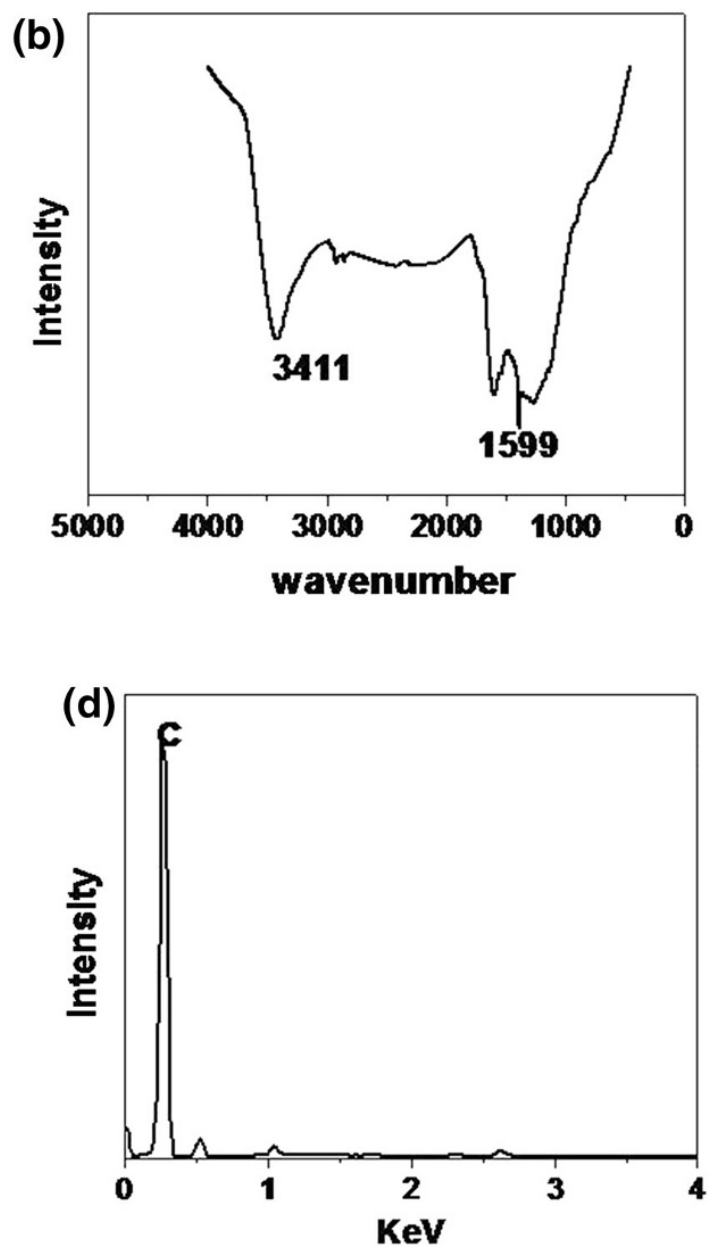

Fig. 1 a Raman spectra of CND showing the characteristic D and G band; b FT-IR of the water soluble CND; c SEM images of water soluble CND; $\mathbf{d}$ EDX of CND showing the major presence of carbon and small amount of oxygen 

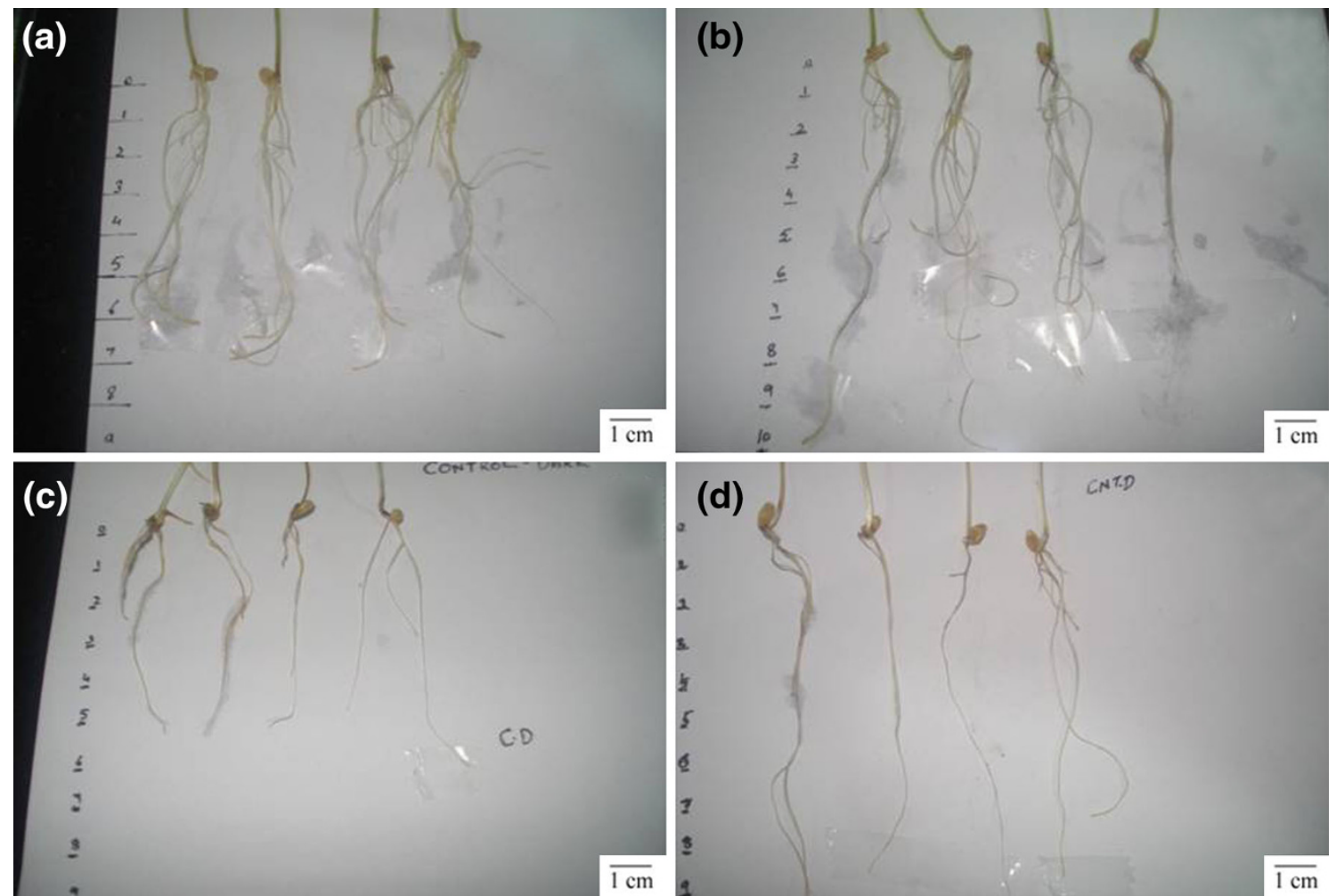

Fig. 2 Phenotypes of wheat roots after 10 days a roots grown only in distilled water under light condition; $\mathbf{b}$ roots grown in wsCND under light condition; $\mathbf{c}$ roots grown only in distilled water under dark condition; $\mathbf{d}$ roots grown in wsCND under dark condition

(a)

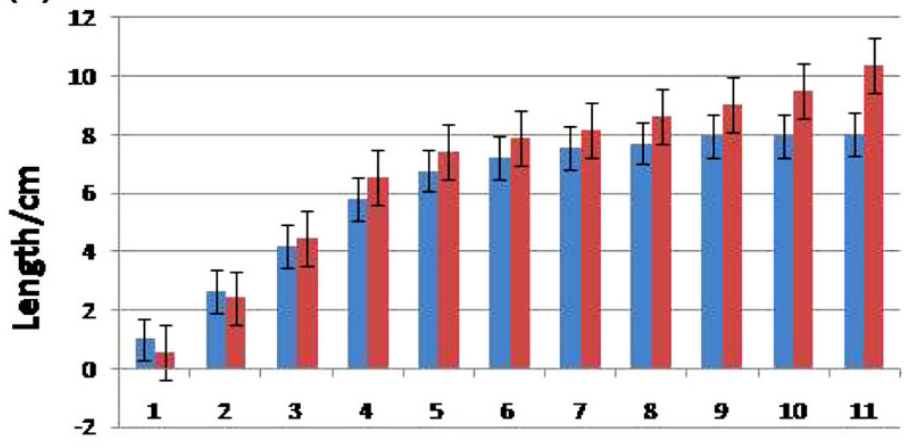

(c)

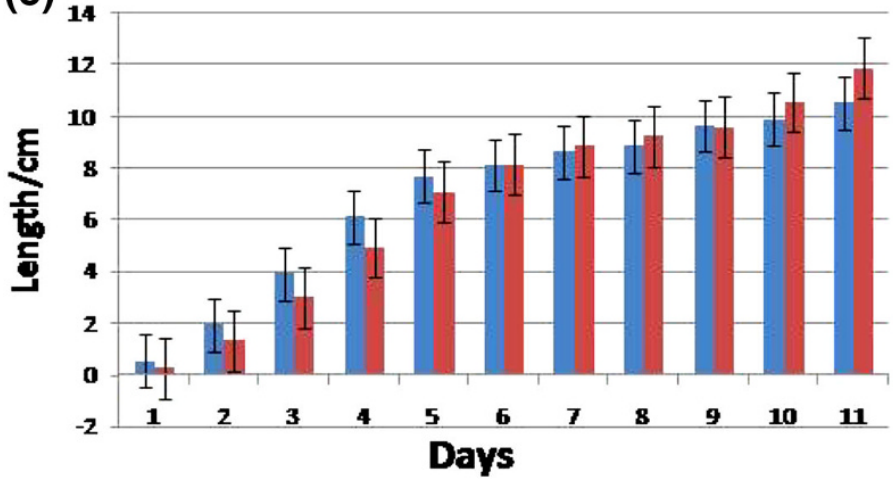

(b)

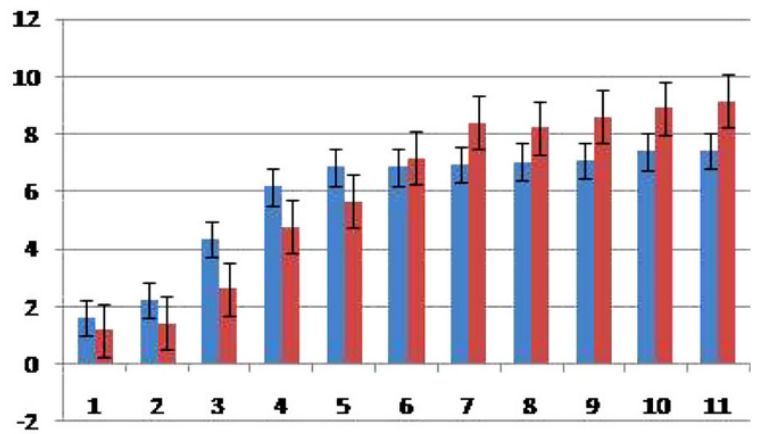

(d)

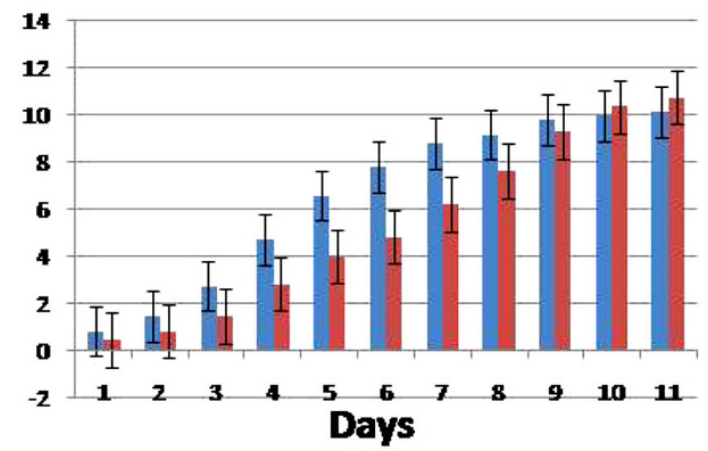

Fig. 3 Histogram comparing the growth of root and shoot with days under different conditions a root in light condition; b root in dark condition; c shoot in light condition; d shoot in dark condition. In this graph blue bars show growth in distilled water and red bars show growth in wSCND 

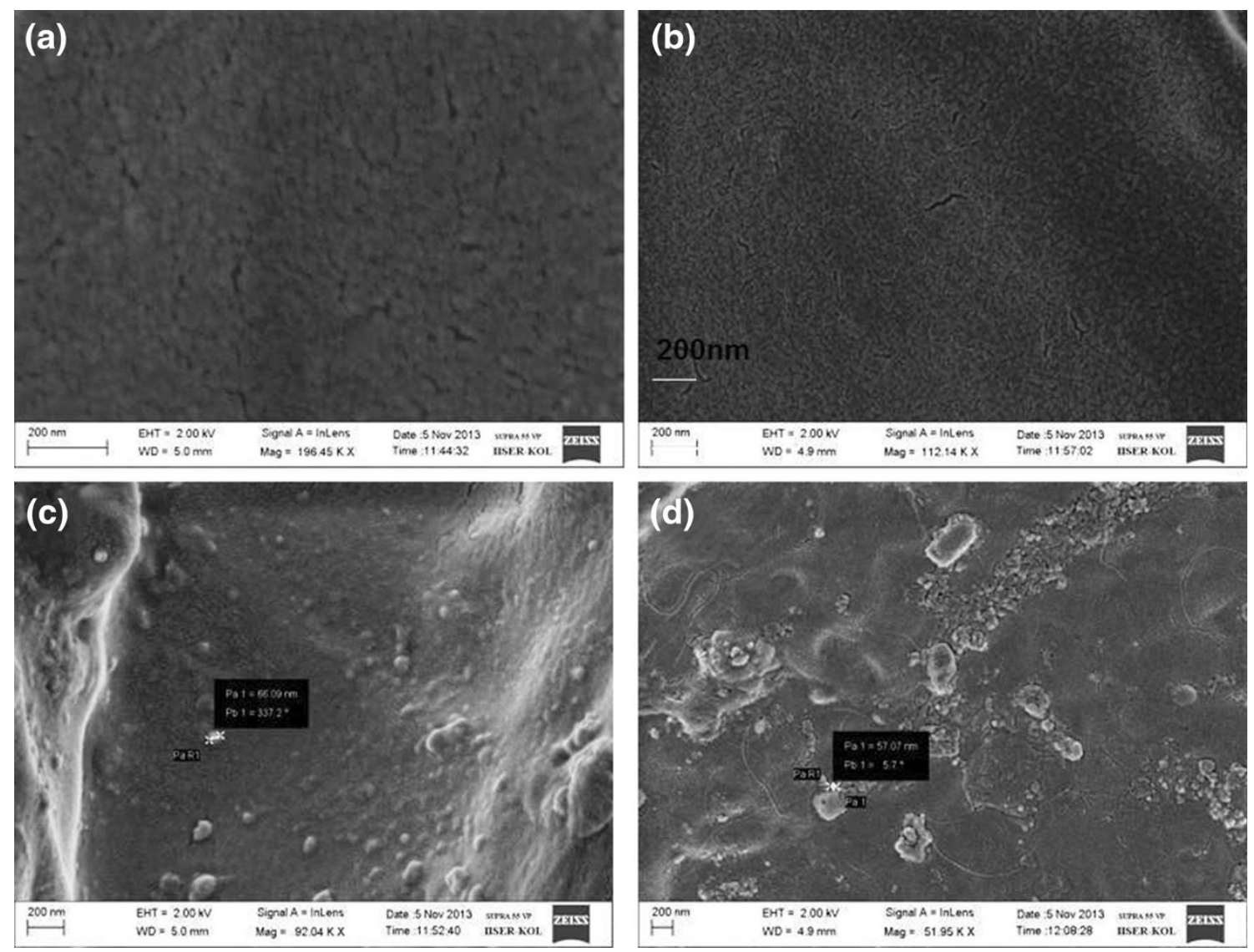

Fig. 4 SEM images of LS of root in light and dark conditions a root grown in distilled water under light condition; $\mathbf{b}$ root grown in distilled water in dark condition; $\mathbf{c}$ root grown in wsCND in light condition; $\mathbf{d}$ root grown in wsCND in dark condition

were grown in distilled water (DW) as well as in solution of wsCND for 10 days. The concentration of wsCND used in the experiment is in the order of $150 \mu \mathrm{g}(200 \mu \mathrm{l})$. The growth of root and shoot of the plant was monitored. A photograph of root grown in DW and in wsCND for 10 days is shown in Fig. 2 (a) growth of root in DW and in wsCND respectively in light condition; (b) growth of root in DW and in wsCND, respectively, in dark condition. It is clear that on average growth of root in wsCND both in light and dark conditions is more compared to that of grown in DW. It is also important to note that growth of root in wsCND under light condition is more compared to that under dark condition. On the other hand, the growth of shoot in wsCND is nearly the same in both light and dark conditions. The effect of wsCND on the growth of shoot was not so visible. Figure 3 compares the growth of shoot in DW and wsCND under light and dark conditions. It is clear that growth of both root and shoot is more in DW for the first few days and after that growth in wsCND becomes more. Such result is expected as seeds at the initial stage house all the essential nutrients inside and these are slowly supplied to nurse the very young sapling at its initial stage. The presence of wsCND at this stage is somewhat not helpful because
wsCND competes to trap nutrients partly hindering the natural growth of the plant sapling at the initial days. However, when the sapling became few days old it is the wsCND which started providing the required nutrients to the sapling slowly as by then the seed exhausted its all nutrients and root and stem of the sapling are formed to harbor wsCND (see below). The growth of root under light condition (Fig. 3a) is more in DW compared to that of in wsCND for first 2 days and after that growth in wsCND becomes more. Under the dark condition, the growth of root (Fig. 3b) in DW is more for the first 5 days and after that growth in wsCND becomes more. The growth of shoot in light (Fig. 3c) and dark (Fig. 3d) conditions is more in wsCND from 6th and 10th day only. It is also important to point out that both Anova Test and Bonferroni (tests for variance) showed that the difference in growth of plant root and shoot in DW and wsCND is significant.

To understand the interaction of wsCND with plants, the SEM images of L. S of roots of the plants grown in DW and in wsCND are shown in Fig. 4. The SEM images for the plants grown in DW under light dark conditions are shown in Fig. 4a and b, respectively. The SEM images for 
Fig. 5 Fluorescence

microscopy image of plant roots

at different duration. In left

panel images for control and in right panel images of wsCND

treated root are shown where the fluorescence due to wsCND can be clearly distinguished from the background auto

fluorescence. Images are with $\times 40$ magnification
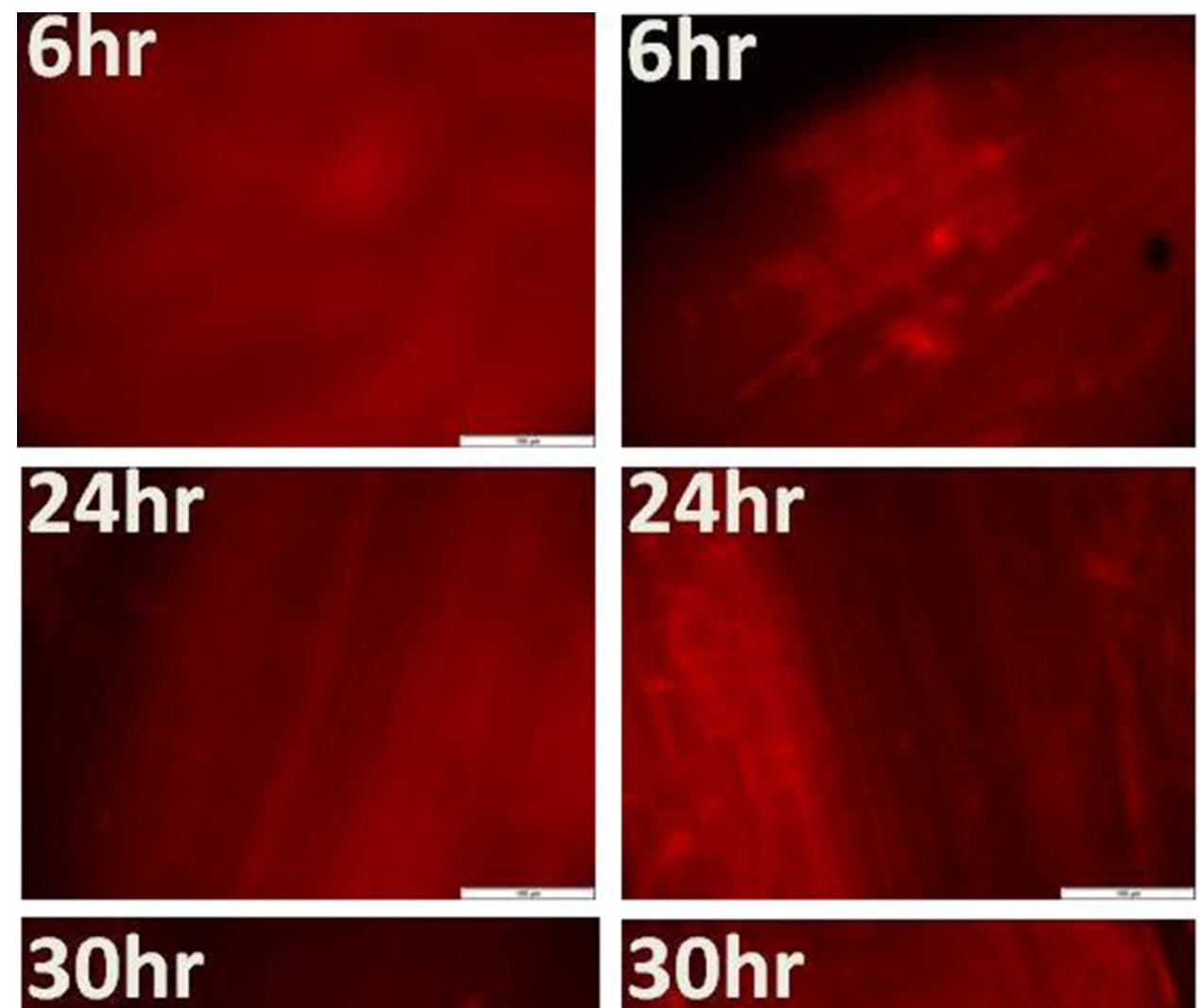

the plant grown in wsCND are shown in Fig. 4c and d, respectively. The subtle difference in Fig. $4 a$ with $c$ and b with d clearly shows that there are interactions between plant roots with wsCND. The wsCND absorbed on the root surface may enter inside the plant body through the pores present on the cell wall and plasmodesmata through apoplastic and symplastic pathways to cross the cortex region. A single layer endodermis may play major role in regulating the movement of water and ions. This endodermis has passage cell which passes water molecules from cortex to protoxylem and may finally reach inside the xylem vessel by apoplastic, symplastic or transmembrane pathway (Clarkson 1993). Once wsCND reaches inside the xylem it is transported to all the parts of the plant body. To further locate the position of wsCND inside the root, we snapped fluorescence images under varied time of its 


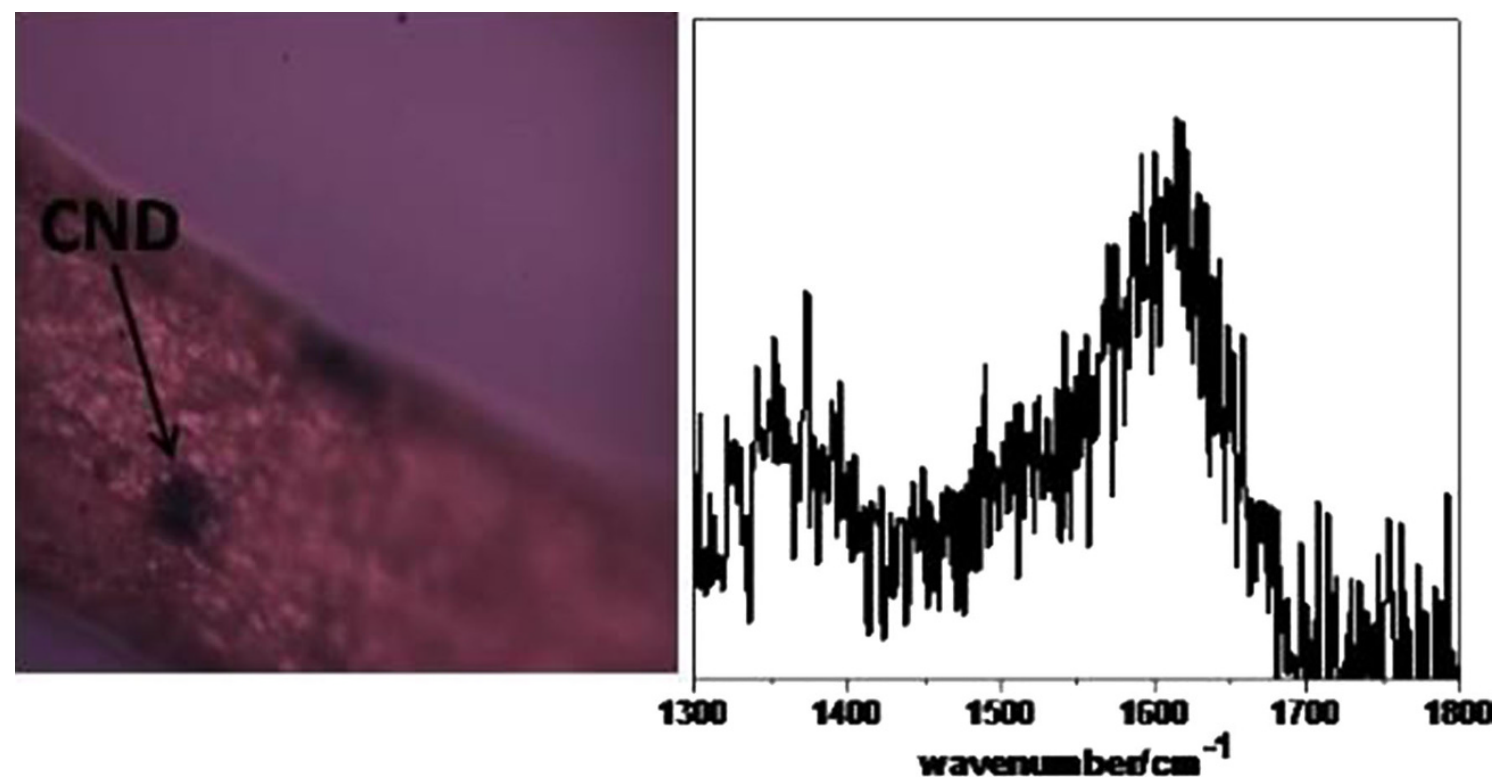

Fig. 6 Raman image and Raman spectrum of plant root treated with wsCND. Arrow in the image shows the presence of wsCND. The Raman spectrum clearly shows the two peaks related to $G$ and $D$ vibrations

uptake like after $6,24,30 \mathrm{~h}$, and finally on the 7 th day. These fluorescence images are shown in Fig. 5. These show that with the increase in time, the movement of the wsCND particle in different regions of the plant follows different pathways, i.e. apolastic or symplastic depending on the diffusion coefficient and transpiration pull. There was no fluorescence in the vascular region of root grown in D. W, whereas fluorescence was observed in the vascular region of the root grown in wsCND. This fluorescence is due to the presence of wsCND. This confirms that wsCND has entered the vascular bundle of the plant. To have more precise location of the CNDs inside the plant, we use Raman image spectroscopy to identify its presence in the root of the plant. This image and Raman spectrum are shown in Fig. 6. This image clearly shows the signature of wsCND which is accumulated in the epidermal and vascular region of the root. The presence of wsCND inside the root can be due their small size and high surface reactivity which allow wsCND to cross the biological barriers.

In summary, we show that water soluble carbon nanodots (wsCND) easily cross the biological barriers in young plant saplings to carry forward more nutrients and water to enhance the growth of wheat plant, one of the most important cereal crops. The healthy growth under wsCND may be related to non-toxic effect of this wsCNDs to wheat plants. Thus growth of wheat plant under such treatment is a good observation as these cereals are rich in carbohydrates, protein, vitamins and minerals.

Acknowledgments S. T. thanks Science and Engineering Research Board (SERB), Department of Science and Technology (DST), India for funding under Fast Track Scheme for Young Scientists. Thanks to Dr. Shree Prakash Pandey for hosting me during the implementation of this project. S. S thanks the DST for a Ramanna Fellowship.

Open Access This article is distributed under the terms of the Creative Commons Attribution License which permits any use, distribution, and reproduction in any medium, provided the original author(s) and the source are credited.

\section{References}

Cañas JE, Long M, Nations S, Vadan R, Dai L, Luo M, Ambikapathi R, Lee EH, Olszyk D (2008) Effects of functionalized and nonfunctionalized single-walled carbon nanotubes on root elongation of select crop species. Environ Toxicol Chem 27:1922-1931. doi:10.1897/08-117.1

Clarkson DT (1993) Roots and the delivery of solutes to the xylem. Philos Trans R Soc B Lond 341:5-17. doi:10.1098/rstb.1993.0086

Feizi H, Moghaddam PR, Shahtahmassebi N, Fotovat A (2012) Impact of bulk and nanosized titanium dioxide (TiO2) on wheat seed germination and seedling growth. Biol Trace Elem Res 146:101-106. doi:10.1007/s12011-011-9222-7

Ghosh M, Sonkar SK, Saxsena M, Sarkar S (2011) Carbon nanoonions for imaging the life cycle of Drosophila Melanogaster. Small 7:3170-3177. doi:10.1002/smll.201101158

Hong F, Zhou J, Liu C, Yang F, Wu C, Zheng L, Yang P (2005a) Effect of nano- $\mathrm{TiO}_{2}$ on photochemical reaction of chloroplasts of spinach. Biol Trace Elem Res 105:269-279. doi:10.1385/BTER: 105:1-3:269

Hong F, Yang F, Liu C, Gao Q, Wan Z, Gu F, Wu C, Ma Z, Zhou J, Yang $\mathrm{P}$ (2005b) Influences of $\mathrm{Nano}^{-\mathrm{TiO}_{2}}$ on the chloroplast aging of spinach under light. Biol Trace Elem Res 104:249-260. doi:10.1385/BTER:104:3:249

Khodakovskaya M, Kim BS, Kim JN, AlimohammadiDervishi ME, Mustafa T, Cernigla CE (2013a) Carbon nanotubes as plant 
growth regulators: effects on tomato growth, reproductive system, and soil microbial community. Small 9:115-123. doi:10.1002/smll.201201225

Khodakovskaya M, Silva KD, Biris AS, Dervishi E, Villagarcia H (2013b) Carbon nanotubes induce growth enhancement of tobacco cells. ACS Nano 6:2128-2135. doi:10.1021/nn204643g

KhodakovskayaM DervishiE, Mahmood M, Xu Y, Li Z, Watanabe F, Biris AS (2009) Carbon nanotubes are able to penetrate plant seed coat and dramatically affect seed germination and plant growth. ACS Nano 3:3221-3227. doi:10.1021/nn302965w

Larue C, Pinault M, Czarny B, Georgin D, Jaillard D, Bendiab N, Mayne-L'Hermite M, Dive MTV, Carrière M (2012) Quantitative evaluation of multi-walled carbon nanotube uptake in wheat and rapeseed. J Hazard Mater 227:155-163. doi:10.1016/j. jhazmat.2012.05.033

Lu CM, Zhang CY, Wen JQ, Wu GR, Tao MX (2002) Research on the effect of nanometer materials on germination and growth enhancement of glycine max and its mechanism. Soybean Sci 21:68-172

Mahajan P, Dhoke SK, Khanna AS (2011) Effect of nano-ZnO particle suspension on growth of mung (Vigna radiata) and Gram(Cicerarietinum) seedlings using plant agar method, pramodmahajan. J Nanotechnol 2011:696535. doi:10.1155/2011/ 696535
Miralles P, JohnsonE Church TL, Harris AT (2012) Multiwalled carbon nanotubes in alfalfa and wheat: toxicology and uptake. J R Soc Interface 9:3514-3527. doi:10.1098/rsif.2012.0535

Sonkar SK, Roy M, Babar DG, Sarkar S (2012) Water soluble carbon nano-onions from wood wool as growth promoters for gram plants. Nanoscale 4:7670-7675. doi:10.1039/C2NR32408C

Tan XM, Lin C, Fugetsu BM (2009) Studies on toxicity of multiwalled carbon nanotubes on suspension rice cells. Carbon 47:3479-3487. doi:10.1016/j.carbon.2009.08.018

Tripathi S, Sonkar SK, Sarkar S (2011) Growth stimulation of Gram (CicerArietinum) plant by water soluble carbon nanotubes. Nanoscale 3:1176-1181. doi:10.1039/C0NR00722F

Wang X, Han H, Liu X, Gu X, Chen K, Lu D (2012) Multi-walled carbon nanotubes can enhance root elongation of wheat (Triticum aestivum) plants. J Nanopart Res 14:841. doi:10.1007/ s11051-012-0841-5

Yang F, Hong F, You W, Chao L, Gao F, Wu C, Yang P (2006) Influence of nano-anatase $\mathrm{TiO}_{2}$ on the nitrogen metabolism of growing spinach. Biol Trace Elem Res 110:179-190. doi:10. 1385/BTER:110:2:179

Yuan H, Hu S, Huang P, Song H, Wang K, Ruan J, He R, Cui D (2011) Single walled carbon nanotubes exhibits dual phase regulations to exposed Arabidopsis Mesophyll cells. Nanoscale Res Lett 6:44. doi:10.1007/s11671-010-9799-3 